\title{
COVID-19 emergency: the influence of implicit attitudes, information sources, and individual characteristics on psychological distress, intentions to get vaccinated, and compliance with restrictive rules
}

\section{BACKGROUND}

To limit the spread of the COVID-19 emergency, a massive vaccination program was implemented and restrictive measures were imposed on the population. However, the propensity to adhere to the vaccination program has struggled to take off. Moreover, complying with the restrictive rules and maintaining social distancing have been highly distressing for many individuals.

\section{PARTICIPANTS AND PROCEDURE}

Italian participants $(N=140$, females $=65 \%$, mean age $=29.50, S D=10.80)$ were presented with an online survey consisting of multiple-choice questions and two single-category implicit association tests (SC-IATs). One SC-IAT evaluated the tendency of participants to automatically associate personal protective equipment (PPE) and vaccines with safety or danger, the other evaluated their tendency to automatically associate social situations with good or bad. Multiple-choice questions explored individual, social, and environmental factors that were expected to contribute to vaccine propensity, compliance with restrictive rules, and feelings of distress.

\section{RESULTS}

Using scientific information sources was related to implicitly associating PPE and vaccines with safety, which in turn was associated with the propensity to get the vaccine. Moreover, being female, young, unsatisfied with social relationships, having suffered health and economic consequences due to the pandemic, and having negative implicit attitudes toward social situations contributed to increasing feelings of distress.

\section{CONCLUSIONS}

Communication may contribute to individuals' behavior and preferences and it can also be associated with implicit attitudes, becoming consequently one of the main leverages to reduce vaccine hesitancy. Recovery programs should prioritize the development of interventions aimed at fostering psychological well-being through the enhancement of social contacts.

\section{KEY WORDS}

vaccine; psychological distress; PPE; COVID-19; implicit attitudes 


\section{BACKGROUND}

Since the end of 2019, the COVID-19 emergency has impacted the world, causing thousands of fatalities and a large number of sick people in all nations (World Health Organization, 2021). Regrettably, the spread of the virus has caused not only serious health problems but also the disruption of individuals' life habits and tremendous harm for many organizations all across the world (Giuntella et al., 2021; Majumdar et al., 2020; Seetharaman, 2020; Wilczyńska et al., 2021). From an economic point of view, the pandemic and the prolonged lockdown periods have caused enormous economic losses for companies and forced individuals to reorganize their job if they had preserved one (Baker et al., 2020; Chudik et al., 2020; Kilic \& Marin, 2020; Radulescu et al., 2021). From a psychological point of view, the research has documented an increase in the feelings of distress with the exponential growth of mental health problems such as depression (BuenoNotivol et al., 2021; Colledani et al., 2021; Pappa et al., 2020; Rehman et al., 2021), anxiety (Santabárbara et al., 2021; Veronese, 2021; Xu et al., 2021), reduced well-being and life satisfaction (Dymecka et al., 2021; Veronese, 2021), insomnia (Kokou-Kpolou et al., 2020; Marelli et al., 2021; Pappa et al., 2020), posttraumatic stress disorder (Fekih-Romdhane et al., 2020; Tang et al., 2020), and use (or abuse) of psychotropic drugs (Zaami et al., 2020). Arguably, social life has been one of the most threatened areas of the individual experience. It was immediately clear that, to limit the spread of the contagion and protect themselves and others, social contacts should have been minimized. Although this easy rule was promptly understood by almost everybody, the rigid norms of isolation and social distancing have been highly distressing for many people, creating psychological difficulties and leaving deep marks on many individuals (Chaturvedi et al., 2021; Türközer \& Öngür, 2020). In a moment of great suffering, people were forced to give up each other, without the possibility to count on the social comfort that, because of the extraordinary condition of uncertainty, dismay, and concern, would have been necessary.

During the pandemic, all media (e.g., social networks, radio, TV, press) have transmitted in unison the need to "keep distance", conveying a clear message: Social contacts are a danger! It should be noted that, while this message has been loud and clear, other communications concerning the pandemic have been largely less coherent. What is the most useful protective personal equipment (PPE)? How is it possible to get the vaccine? How should one behave in the case of contagion? How can people cope with feelings of discomfort? The answers to these questions have often been communicated in inconsistent and unscientific ways and, sometimes, with the spread of fake news (Kim \& Kreps, 2020; Moscadelli et al., 2020; Nguyen et al., 2020). This generated anxiety and contributed to making health promotion messages less effective and accepted by the population. For instance, even though the population, in general, understood the need to use masks, sanitizing gels, and other PPE, the propensity to adhere to vaccination programs has struggled to take off (Abu-Farha, 2021; Sallam, 2021; Troiano \& Nardi, 2021). Moreover, also the compliance with the use of PPE was not as widespread as one might have expected (Haischer et al., 2020; Mallinas et al., 2021). For instance, it was not uncommon that people refused or forgot to use specific PPE. Also among those populations with increased risk of contagion or especially exposed to its most adverse consequences (such as the elderly and healthcare professionals), there were non-negligible rates of vaccine hesitancy and irregular use of PPE (Biswas et al., 2021a; Chan et al., 2021; Kose et al., 2021; Reno et al., 2021).

Probably, the good disposition toward the vaccination and the compliance with the rules to limit the spread of the contagion have been influenced by numerous individual, social, and environmental aspects (Murphy et al., 2021; Nazlı et al., 2021; Robertson et al., 2021). Understanding how these factors impacted the willingness to get vaccinated and the compliance with the rules for fighting the virus represents a valuable research objective that may help to improve their acceptance. Other crucial aspects that deserve research investigation concern the negative effects inflicted on individuals and their psychosocial well-being by social isolation. This can lead to the development of more tailored and effective recovery programs.

\section{AIMS AND HYPOTHESES OF THE STUDY}

This study has two aims. The first is to investigate the association of several environmental, social, and individual factors with the willingness of people to get vaccinated and comply with the rules aimed at limiting the spread of the contagion. The following factors are considered: the role of specific information sources (i.e., traditional media, social networks, personal communications, and scientific sources), the impact of pandemic-related variables (i.e., the perception of having suffered health, economic, psychological, or occupational consequences due to the pandemic; the severity of the pandemic situation in one's own home area), and the role of individual differences (i.e., gender, age, personality traits), and attitudes. Concerning attitudes, the aim is to investigate people's perceptions of vaccines and PPE. For this purpose, an implicit measure is used that allows for investigating the extent to which people automatically perceive PPE and vaccines as positive objects associated with safety or as phobic stimuli associated with danger. The hypothesis is that associating vaccines and PPE with safety contributes to greater 
acceptance of them, while associating these objects with danger reduces their acceptance (Hypothesis 1). The influence of information sources on the development of implicit attitudes is also explored. In particular, it is hypothesized that using scientific information sources (e.g., scientific reports or academic papers) is associated with the development of positive attitudes and greater acceptance of vaccines and PPE, while using less scientific sources (e.g., traditional media such as TV and radio, social networks such as Twitter or Facebook, personal communications such as word of mouth), where fake news is more frequent (Fernández-Torres et al., 2021), is associated with the development of negative attitudes and reduced acceptance of vaccines and PPE (Hypothesis 2). The possible mediating effect of implicit attitudes on the relationship between the use of specific information sources and the acceptance of PPE and vaccines is explored as well. In particular, it is hypothesized that using scientific information sources promotes greater acceptance of vaccines and PPE by fostering positive attitudes toward them (i.e., associating them with safety), while relying on unscientific sources promotes lower acceptance of vaccines and PPE by stimulating negative attitudes toward them (i.e., associating them with danger; Hypothesis 3).

The second aim of the work is to investigate the influence of environmental, social, and individual factors on the psychological feelings of distress experienced by people during the pandemic. To achieve this second goal, the following elements are considered: pandemic-related variables, information sources, and individual characteristics such as demographics, personality traits, satisfaction with social relationships, and attitudes toward social situations. Concerning this latter variable, it is expected that in the pandemic time some people might manifest an ambivalent attitude toward social situations. Indeed, people may live in conflict: On the one hand, they feel the need for social contacts, while on the other hand, they are scared of them. It is expected that the concern for the contagion and the need to keep distance from others may lead some individuals to implicitly associate social situations with an event that may cause harm rather than with a positive event that may give comfort. To evaluate this possibility, an implicit measure is used that aims at exploring the extent to which people associate social situations with good or bad. The hypothesis is that manifesting a negative implicit attitude toward social situations is associated with a reduction in well-being (Hypothesis 4).

The attitudes toward social situations and PPE and vaccines are investigated using implicit measures since they make it possible to reveal feelings and attitudes of which people may not be fully aware. Moreover, compared with explicit measures, they are also less affected by social desirability (Colledani \& Camperio Ciani, 2021; Greenwald et al., 1998; Karpinski
\& Hilton, 2001; Nosek, 2007; Steffens, 2004). In addition, it is well known that implicit attitudes, together with explicit ones, strongly contribute to shaping individuals' behaviors and subjective feelings (Banse et al., 2015; Greenwald et al., 2009).

\section{PARTICIPANTS AND PROCEDURE}

\section{PARTICIPANTS}

One hundred and forty respondents were included in the study. Participants were 19 to 65 years old (mean age $=29.50, S D=10.80)$ and $65 \%$ were female $(N=91)$. The majority of respondents were from Northern Italian regions $(71.4 \%$, South of Italy $-8.6 \%$, central Italy $-20 \%$ ) and had a university degree (65\%, high school diploma $-30 \%$, basic education $-5 \%$ ). Among them, $50.7 \%$ were people who were not working (i.e., unemployed, students, housewives, retired persons), $23.6 \%$ were office workers, $7.1 \%$ were intellectual workers (e.g., teachers), 3.6\% were manual workers, $2.9 \%$ were managers or entrepreneurs, $2.1 \%$ were healthcare professionals, and $10 \%$ were workers from other unspecified sectors.

\section{PROCEDURE}

All respondents were enrolled through convenience sampling to fill out an electronic survey. Their participation in the study was anonymous and voluntary. Before accessing the survey, all participants were required to agree by way of an electronic informed consent form that detailed the aims of the study, the task duration, and the possibility of withholding consent to participate in the research at any time. Data were collected between February and May 2021. All ethical standards were respected following the principles of the Declaration of Helsinki.

\section{MEASURES}

Participants answered a questionnaire including a set of measures aimed at investigating individual characteristics, subjective feelings, and usual behaviors. Moreover, they were required to perform two single category implicit association tasks (SC-IAT; Karpinski \& Steinman, 2006) aimed at exploring their implicit attitudes toward vaccines and PPE, and social situations. After a few questions concerning demographic details, participants performed the first SC-IAT. Then they were invited to answer some multiple-choice items and, finally, they performed the second SC-IAT. The order of presentation of the two SC-IATs was randomized among participants (some of them completed first the SC-IAT concerning social
COVID-19: implicit attitudes, vaccine and well-being 
situations and thereafter that concerning PPE and vaccines, whereas other participants performed the two tasks in the opposite order).

\section{Explicit measures}

Two six-item scales of the Eysenck Personality Questionnaire Revised-Abbreviated were used to evaluate extraversion and neuroticism (Colledani et al., 2019). The items were dichotomously scored (i.e., yes or no). In the current sample, the two scales had satisfactory Cronbach's $\alpha$ coefficients (.62 and .77 for neuroticism and extraversion, respectively).

Four items were administered to investigate the severity of the consequences that respondents experienced at health, economic, psychological, and work or study levels due to the pandemic (e.g., "Please indicate how serious the psychological consequences you have suffered because of the pandemic are"). For these items, the answer format was on a six-point scale from 1 (very slight or not at all) to 6 (very serious). Moreover, respondents were asked to report how severe the pandemic situation was in their home area (i.e., "How serious is or has been the pandemic situation in your area of residence?"). This item was answered on a 4-point scale from 0 (not at all) to 3 (very much). An additional item was administered to investigate the extent to which respondents felt the need to obtain professional psychological support during the pandemic (i.e., "Did you feel the need for professional psychological support during the pandemic?"). This item was scored on a 4-point scale from 0 (not at all) to 3 (very much). This item was averaged with that pertaining to the perception of having suffered psychological consequences due to the pandemic to obtain a composite measure of psychological distress.

Two items were administered to investigate the intention to get vaccinated ("Please indicate your preference about the COVID-19 vaccines") and the compliance with the rules to fight the virus ("Please indicate how much you respected the rules for containing the spread of the contagion [e.g., using face masks, respecting social distancing, using sanitizing gel]"). The first item was scored on a seven-point scale from 1 (I don't want to get vaccinated) to 7 (I've already been vaccinated), while the second was scored on a six-point scale from 1 (rarely or never) to 6 (always or almost always).

Four items were administered to investigate the frequency of use (i.e., "How often did you use the following communication channels to obtain information during the pandemic?") of the following information sources: social networks (e.g., Twitter messages or Facebook posts), scientific sources (e.g., scientific articles, scholarly papers), traditional media (e.g., TV, newspapers, radio), and personal communications (e.g., word of mouth). These items were scored on a six-point scale from 1 (rarely or never) to 6 (very often).

Satisfaction with social relationships was investigated through three items (e.g., "I feel satisfied with my social relationships") scored on a four-point scale from 1 (strongly disagree) to 4 (strongly agree). In the current sample, the $\alpha$ coefficient was satisfactory $(\alpha=.72)$.

\section{Implicit measures}

Personal protective equipment - single category implicit association test (PPE-SC-IAT) and social situations - single category implicit association test (SS-SC-IAT). Two SC-IATs were used to obtain an implicit measure of the attitudes of respondents toward PPE and social situations. In these computerized tasks, participants were required to categorize a set of stimuli (words or pictures) presented in the center of the computer screen into the categories displayed at the top-left and top-right screen corners by pressing one of the two indicated response keys (I or E) as quickly and accurately as possible. In the PPE-SC-IAT, the categories were: PPE (represented by five pictures of, for instance, masks, COVID vaccine, sanitizing gel; the picture stimuli are available upon request from the first author), danger (represented by five words: "risky", "emergency", "threat", "uncertainty", "vulnerability"), and safety (represented by five words: "security", "protection", "assurance", "safe", "immunity”). For the SS-SC-IAT, the categories were: social situations (represented by five pictures displaying, for instance, a dinner with friends, a child interacting with grandparents, meetings with friends; the picture stimuli are available upon request from the first author), good (represented by five words: "pleasure", "life", "positive”, "nice”, "lovely"), and bad (represented by five words: "worry", "stress", "contagion", "anxiety", "death"). The idea behind the SC-IAT is that the categorization task should be easier (faster and more accurate responses) when the two concepts that for an individual are implicitly associated are mapped with the same response key. Conversely, the categorization task should be more difficult (slower and less accurate responses) when these concepts are mapped with a different response key. For example, for people who consider social situations as positive events, the responses are assumed to be faster and more accurate when the categories social situations and good are mapped onto the same key than when social situations and bad are mapped onto the same key (Karpinski \& Steinman, 2006).

The SC-IATs used in the present work consisted of two practice and two test blocks. The practice blocks included 20 trials each and the test blocks consisted of 60 trials each. Each block was preceded by a set of instructions and, to avoid effects due to the order of presentation, the blocks were balanced over par- 
ticipants (i.e., some participants had to complete first the block in which PPE and safety stimuli shared the same response key, while others had to complete first the block in which the same response key was shared by PPE and danger).

\section{ANALYSIS}

Descriptive statistics were computed for all the used variables. The D-scores at the two SC-IATs were calculated according to the indications in the literature (Karpinski \& Steinman, 2006). In particular, they were computed as the difference between the average response time in the two test blocks standardized by the standard deviation of the correct trials in these blocks. Responses faster than $350 \mathrm{~ms}$ and non-responses (i.e., responses exceeding 1,500 ms) were replaced by the average response time of the block in which the error occurred, increased by a 400 ms penalty. When there were $25 \%$ or more incorrect responses in at least one of the critical blocks, the D-scores were taken to be "out of accuracy" (Epifania et al., 2020) and were not considered for further analyses. The D-scores were computed so that a positive value indicated a positive implicit attitude toward the object (i.e., PPE are associated with safety; social situations are associated with good) while a negative value indicated a negative attitude toward the object (i.e., PPE were associated with danger, social situations were associated with $b a d$ ). D-scores were obtained using the R package ImplicitMeasures (Epifania et al., 2020). D-scores of $0.15,0.35$, and 0.65 in absolute values were considered as "slight", "moderate", and "strong" effects, respectively.

To test the research hypotheses, three regression models were run using the backward method. In the first model, the dependent variable was the score on the item investigating the compliance with the rules aimed at limiting the spread of the contagion. In the second model, the dependent variable was the score on the item pertaining to the intention of getting vaccinated. In both models, the independent variables were: the D-score at the PPE-SC-IAT (Hypothesis 1), the frequency of use of the different information sources (i.e., scientific, personal communications, traditional media, and social networks; Hypothesis 2), a set of pandemic-related variables (the severity of the consequences suffered at health, economic, psychological, and occupational levels; the severity of the pandemic situation in the home area), and individual and demographic characteristics such as age, gender, and personality traits (i.e., neuroticism and extraversion). The influence of the considered information sources on implicit attitudes toward vaccines and PPE was explored in a separate regression model (Hypothesis 2). The possible mediating effect of implicit attitude toward PPE and vaccines on the relationship between information sources and outcome variables (Hypothesis 3) was explored using Mplus 7.4 (Muthén \& Muthén, 2012) and the maximum likelihood estimator. The statistical significance of the indirect effect was verified using a bootstrapping method (5,000 resamples and 95\% bias-corrected confidence interval).

The third regression model was run to explore the influence of a set of variables on the feelings of psychological distress experienced by people during the pandemic. In this model, the independent variables were: the D-score at the SS-SC-IAT (i.e., implicit attitude toward social situations; Hypothesis 4), the frequency of use of the different information sources (i.e., scientific, personal communications, traditional media, and social networks), pandemic-related variables (i.e., the severity of health, work-study, and economic consequences suffered due to the pandemic; the severity of the pandemic situation in the home area), and individual and demographic characteristics (i.e., age, gender, satisfaction with social relationships, neuroticism, and extraversion).

\section{RESULTS}

Descriptive statistics for the used variables are reported in Table 1. The results showed that a large part of respondents played by the rules and used PPE always or almost always (47.7\%). The intention to get vaccinated was also rather high, with $70 \%$ of respondents reporting being vaccinated already or strongly willing to get the vaccine. Conversely, $30 \%$ of respondents reported being hesitant toward vaccination $(7.1 \%$ were strongly hesitant). The results of the PPE-SC-IAT indicated that $22.6 \%$ of respondents implicitly associated PPE and vaccines with danger, while only $11.3 \%$ associated them with safety (with a moderate to strong effect; the D-scores of 16 respondents were excluded because they were "out of accuracy"). Likewise, the SS-SC-IAT indicated that $28.3 \%$ of respondents implicitly associated social situations with bad, while only $14.5 \%$ associated them with good (with a moderate to strong effect; the D-scores of only two respondents were excluded because they were "out of accuracy").

Table 2 reports the results of the backward regression model aimed at exploring the influences of a set of social, environmental, and individual variables on compliance with the rules aimed at limiting the spread of the contagion (and the actual use of PPE). Only two out of the 14 variables inserted in the initial step of the analysis showed significant effects $\left(R^{2}=.06, F(2,121)=3.74, p=.026\right)$. In particular, the compliance with the rules to fight the virus and the use of PPE were higher among females and lower among people who mainly relied on personal communications to keep informed (Hypothesis 2).
COVID-19: implicit attitudes, vaccine and well-being 
Table 1

Daiana Colledani, Pasquale Anselmi, Egidio Robusto

Descriptive statistics for all the used variables

\begin{tabular}{lccc}
\hline & $N$ & $M$ & $S D$ \\
\hline D-score PPE-SC-IAT & 124 & -0.08 & 0.36 \\
D-score SS-SC-IAT & 138 & -0.10 & 0.38 \\
Intentions to get vaccinated & 140 & 4.98 & 1.42 \\
Respect of restrictive rules and use of PPE & 140 & 5.24 & 0.90 \\
Severity of the pandemic in the home area & 140 & 1.74 & 0.72 \\
Extraversion & 140 & 0.45 & 0.34 \\
Neuroticism & 140 & 0.51 & 0.29 \\
Need for professional psychological support & 140 & 1.09 & 1.03 \\
Psychological distress (composite measure) & 140 & 2.35 & 1.13 \\
Satisfaction with social relationships & 140 & 2.73 & 0.55 \\
Health consequences due to the pandemic & 140 & 1.78 & 1.29 \\
Economic consequences due to the pandemic & 140 & 2.71 & 1.78 \\
Work/study consequences due to the pandemic & 140 & 3.35 & 1.71 \\
Psychological consequences due to the pandemic & 140 & 3.61 & 1.47 \\
Frequency of use of scientific information sources & 140 & 3.15 & 1.55 \\
Frequency of use of social networks as information sources & 140 & 3.52 & 1.68 \\
Frequency of use of traditional media as information sources & 140 & 4.24 & 1.67 \\
Frequency of use of personal communication as information sources & 140 & 3.34 & 1.42 \\
\hline Note PPE-SC-IAT personal
\end{tabular}

Note. PPE-SC-IAT - personal protective equipment - single category implicit association test; SS-SC-IAT - social situations - single category implicit association test.

Table 2

Backward regression model with respect of restrictive rules and use of PPE as the dependent variable

\begin{tabular}{lrrrrr}
\hline & $B$ & $S E$ & $\beta$ & $t$ & $p$ \\
\hline Intercept & 5.35 & 0.21 & & 25.90 & $<.001$ \\
Gender (females - 1, males - 0) & 0.38 & 0.17 & .21 & 2.29 & .024 \\
Personal communications & -0.11 & 0.06 & -.19 & -2.03 & .045 \\
\hline
\end{tabular}

Note. PPE - personal protective equipment; $B$ - unstandardized coefficients; $\beta$ - standardized coefficients; $R^{2}=.06, F(2,121)=3.74$, $p=.026$.

Table 3

Backward regression model with intentions to get vaccinated as the dependent variable

\begin{tabular}{lccccc}
\hline & $B$ & $S E$ & $\beta$ & $t$ & $p$ \\
\hline Intercept & 4.15 & 0.33 & & 12.71 & $<.001$ \\
Extraversion & 0.80 & 0.36 & .19 & 2.23 & .028 \\
Scientific information sources & 0.18 & 0.08 & .20 & 2.24 & .027 \\
D-score at the PPE-SC-IAT & 0.80 & 0.34 & .20 & 2.33 & .022 \\
\hline
\end{tabular}

Note. PPE-SC-IAT - personal protective equipment - single category implicit association test; $B$ - unstandardized coefficients; $\beta$ - standardized coefficients; $R^{2}=.13, F(3,120)=5.94, p=.001$. 
The model exploring the influence of the 14 variables on the intentions to get vaccinated showed a different result. In this model, three variables were found to be relevant in influencing vaccine propensity, and implicit attitudes were among them $\left(R^{2}=.13\right.$, $F(3,120)=5.94, p=.001$; Table 3$)$. In particular, the results indicated that extraverted people, individuals who used scientific sources, and those who implicitly associated PPE and vaccines with safety were more willing to be vaccinated (Hypotheses 1 and 2).

According to Hypothesis 2, the regression model exploring the influence of information sources (i.e., scientific information sources, personal communications, traditional media, and social networks) on implicit attitudes revealed that using scientific sources was associated with a positive implicit attitude toward vaccines and PPE $(\beta=.22, p=.016)$. Conversely, the use of the other considered information sources was found not to be related to implicit attitudes.

Considering these results, a mediation model was tested in which the use of scientific information sources was set as the independent variable, the $\mathrm{D}$-score at the PPE-SC-IAT was the mediating vari- able, and the propensity to get vaccinated was the dependent variable. In line with Hypothesis 3, the results of this model supported the mediating effect of implicit attitudes toward PPE on the relationship between the use of scientific information sources and vaccine propensity (Figure 1). Specifically, the model suggested that the more people took information from scientific sources, the more they were inclined to implicitly associate PPE with safety and, consequently, the more they were favorable towards getting vaccinated. A positive direct effect between scientific information sources and vaccine propensity was also observed.

Table 4 reports the results of the backward regression model that explored the influence of social, environmental, and individual variables on the subjective feelings of distress experienced by people during the pandemic (i.e., perception of having suffered psychological consequences and need for professional psychological support). Six out of the 14 variables included in the analysis provided a significant contribution to the explanation of psychological distress $\left(R^{2}=.43, F(6,131)=16.69, p<.001\right)$. Specifically, the

\section{Figure 1}

Unstandardized (and standardized) coefficients in the mediation model

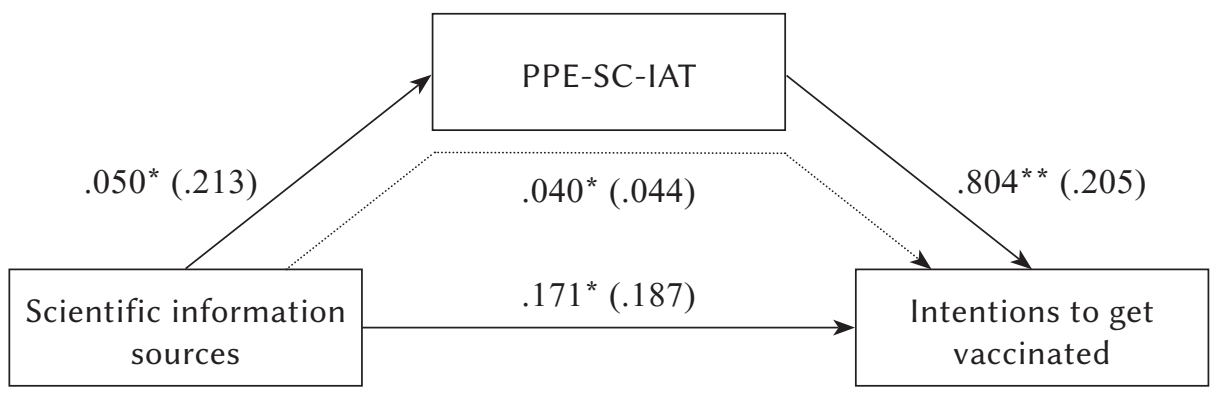

Note. PPE-SC-IAT - personal protective equipment - single category implicit association test. The dotted line represents the indirect effect. ${ }^{*} p<.05,{ }^{* *} p<.01$.

Table 4

Backward regression model with psychological distress as the dependent variable

\begin{tabular}{lrrrrr}
\hline & $B$ & $S E$ & $\beta$ & $t$ & $p$ \\
\hline Intercept & 4.00 & 0.50 & & 7.97 & $<.001$ \\
Age & -0.04 & 0.01 & -.38 & -5.71 & $<.001$ \\
Gender (females - 1, males - 0) & 0.39 & 0.16 & .16 & 2.43 & .017 \\
Health consequences & 0.16 & 0.07 & .18 & 2.47 & .015 \\
Economic consequences & 0.16 & 0.05 & .25 & 3.33 & .001 \\
Satisfaction with social relationships & -0.54 & 0.14 & -.27 & -3.95 & $<.001$ \\
D-score SS-SC-IAT & -0.43 & 0.20 & -.14 & -2.16 & .032 \\
\hline
\end{tabular}

Note. SS-SC-IAT - social situations - single category implicit association test; $B$ - unstandardized coefficients; $\beta$ - standardized coefficients; $R^{2}=.43, F(6,131)=16.69, p<.001$. 
results indicated that females and younger people experienced higher distress. Moreover, positive associations were observed between feelings of distress and the perception of having suffered health and economic consequences due to the pandemic. Feelings of distress were found to be negatively associated with implicit attitudes toward social situations (Hypothesis 4) and satisfaction with social relationships.

Daiana Colledani,

Pasquale Anselmi, Egidio Robusto

This study aimed at investigating two aspects that have crucial relevance in influencing the course and the future consequences of the COVID-19 emergency. In particular, the study explored how individual characteristics, implicit attitudes, information sources, and variables associated with the pandemic condition have contributed to the propensity of individuals to take actions for limiting the spread of the contagion (i.e., using PPE and respecting restrictive rules, getting vaccinated) and their psychosocial well-being.

Overall, the respondents declared that they were careful in using PPE and respectful of restrictive rules. However, a significant role of individual characteristics and social factors was observed in these behaviors. In particular, males and people who relied mainly on personal communications to obtain information demonstrated lower compliance with the rules. Information sources and individual characteristics provided a relevant influence also concerning the propensity toward vaccination. Although the percentage of people willing to be vaccinated (or already vaccinated) was quite high (over $70 \%$ ), in the present study, a non-negligible $7.1 \%$ of respondents reported being strongly hesitant or against the vaccine. This percentage rises to $30 \%$ when also including mild hesitancy. This percentage is in line with other studies in Italy (e.g., Reno et al., 2021) and around the world (Aw et al., 2021; Biswas et al., 2021b; Sallam, 2021), despite the extensive vaccination campaigns and the availability of alternative vaccines (Haynes, 2021; Pawliczak, 2021). The variables that mainly contributed to this attitude were: personality traits (i.e., extraversion), the positive implicit attitude towards PPE and vaccines (Hypothesis 1), and the use of scientific information sources (Hypothesis 2). In particular, it was found that associating PPE and vaccines with safety instead of danger was positively related to the willingness to get vaccinated. However, this type of association was not as common as one might have expected. In fact, only $11.3 \%$ of participants moderately to strongly associated them with safety, while $22.6 \%$ showed an opposite attitude, moderately to strongly associating them with danger. According to the research hypotheses, the use of scientific information sources was found to sig- nificantly contribute to this attitude (Hypothesis 2). Specifically, preferring scientific sources has been found to be related to the tendency of individuals to associate PPE and vaccines with safety that, in turn, is associated with a greater propensity of individuals to get vaccinated (Hypothesis 3). These results are in line with Hypotheses 1, 2, and 3. Taken together, they highlight that, while scientific information sources (e.g., scientific reports or academic papers) are associated with the development of positive attitudes and greater acceptance of vaccines and PPE, unscientific communications (i.e., word of mouth) - that are often affected by the spread of inaccurate or fake news (Fernández-Torres et al., 2021) - are associated with reduced acceptance of them. This indicates the crucial role of communication in the pandemic time. Communication may contribute to individuals' behavior and preferences, and it can also be associated with the most profound attitudes and feelings of individuals, becoming consequently one of the main leverages that can be used to reduce vaccine hesitancy.

Implicit attitudes and individual and social characteristics also turned out to be central factors concerning the second topic addressed by this study: psychosocial well-being in the time of emergency. The results indicated that feelings of distress were associated with individual characteristics such as gender and age (females and younger people were more exposed to feelings of distress), with the perception of having suffered serious economic and health consequences due to the pandemic, and with the dissatisfaction with social relationships. This result is not surprising given the crucial role of individual differences in coping with the distress associated with the pandemic condition (Glowacz \& Schmits, 2020; Guo et al., 2021; Merlo et al., 2021; Schou-Bredal et al., 2021; Xiong et al., 2020; Yıldırım \& Güler, 2021) and the severe limitations that people have to face to limit the spread of the contagion. However, it is more interesting that psychological well-being was associated not only with the dissatisfaction of people with their social relationships but also with their implicit negative attitude toward social situations in general (Hypothesis 4). The role of implicit attitudes toward social situations has been explored because it was hypothesized that, during the pandemic, the concerns for the contagion and the need to keep distance from others may have led some individuals to implicitly associate these events with a condition that may cause harm rather than with a positive situation that may give comfort. The results of the study suggest that this has been the case. In fact, $28.3 \%$ of respondents associated social situations with $\mathrm{bad}$, while only a lower $14.5 \%$ associated them with good. This suggests that, at present, people may manifest ambivalent and not fully positive feelings toward social situations. On the one hand, they may feel the desire to experience connection and so- 
cial support but, on the other hand, they are afraid of contagion and inhibited in their relationships by the restrictions imposed by the pandemic. At this time, individuals cannot rely on social comfort but are, on the contrary, frightened of it, and this has a strong negative impact on their well-being. While having facilitated control over the contagion, social distancing has also had negative effects on other aspects of human life. Taking into account these findings, future recovery programs should be addressed to developing interventions aimed at helping people to regain confidence in social situations. Recovery programs, in other words, should be aimed at healing the wounds caused, at the social level, by the necessary social distancing policies. According to the literature, these interventions should be addressed to the whole population and mainly to the social groups that have experienced greater difficulties, such as young people, disabled people and their families, females, and health care professionals (Batra et al., 2020; Glowacz \& Schmits, 2020; Guo et al., 2021; Khasawneh, 2021; Ranieri et al., 2021; Xiong et al., 2020).

Overall, the merit of this work was that it addressed two key issues in the management of the current pandemic and that may also have effects in the future. The use of implicit measures was a second significant advantage. These measures allow for overcoming the difficulties associated with social desirability and with the limited introspection of individuals, which represent threats that can impair the validity of psychosocial research. Implicit measures capture profound feelings of which people may not be fully aware and, according to the literature, they are significantly associated with individuals' behaviors (Banse et al., 2015; Greenwald et al., 1998, 2009; Richetin et al., 2007, 2010). Moreover, in this study, implicit attitudes were found to have a strong impact on individuals' well-being and behaviors, also when controlling for the influence of other relevant individual variables (e.g., personality traits).

The findings presented in this paper may provide interesting insight not only concerning the management of the pandemic but also concerning the general topic of the promotion of safety in several fields. To improve the acceptance of safety practices, a central role should be given to scientific information that, by promoting positive attitudes toward them, increases their acceptance. These findings can be easily extended to the fields of road and workplace safety. For this purpose, it could be suggested to make scientific communication as clear, accessible, and suitable for different audiences as possible. This is expected to limit the spread of communications that are simplified, often inaccurate, and with a negative effect on compliance with safety rules.

Even though the results of this study are promising and useful to better understand how people coped with the challenges deriving from the spread of the pandemic, some limitations could be pointed out. Two of them are the small sample size and the cross-sectional nature of the study. To provide further support for our findings, studies in other cultural contexts, with longitudinal designs and with larger samples, are advocated.

In this study, explicit attitudes were not considered because the main objective of the study was the investigation of automatic attitudes and not fully controlled reactions of individuals toward vaccines and PPE and toward social situations. Future studies should also consider explicit measures of attitudes because of the incremental contribution they can provide in the explanation of behavior and individuals' preferences. These studies may provide further insights for the development of appropriate and effective interventions.

\section{RefERENCES}

Abu-Farha, R., Mukattash, T., Itani, R., Karout, S., Khojah, H., Al-Mahmood, A., \& Alzoubi, K. (2021). Willingness of Middle Eastern public to receive COVID-19 vaccines. Saudi Pharmaceutical Journal, 29, 734-739. https://doi.org/10.1016/j.jsps. 2021.05.005

Aw, J., Seng, J. J. B., Seah, S. S. Y., \& Low, L. L. (2021). COVID-19 vaccine hesitancy - a scoping review of literature in high-income countries. Vaccines, 9, 900. https://doi.org/10.3390/vaccines9080900

Baker, S. R., Bloom, N., Davis, S. J., \& Terry, S. J. (2020). COVID-induced economic uncertainty. National Bureau of Economic Research. Retrieved from https://www.nber.org/papers/w26983

Banse, R., Messer, M., \& Fischer, I. (2015). Predicting aggressive behavior with the aggressivenessIAT. Aggressive Behavior, 41, 65-83. https://doi. org/10.1002/ab.21574

Batra, K., Singh, T. P., Sharma, M., Batra, R., \& Schvaneveldt, N. (2020). Investigating the psychological impact of COVID-19 among healthcare workers: a meta-analysis. International Journal of Environmental Research and Public Health, 17, 9096. https://doi.org/10.3390/ijerph17239096

Biswas, M., Alzubaidi, M. S., Shah, U., Abd-Alrazaq, A. A., \& Shah, Z. (2021a). A scoping review to find out worldwide COVID-19 vaccine hesitancy and its underlying determinants. Vaccines, 9, 1243. https://doi.org/10.3390/vaccines9111243

Biswas, N., Mustapha, T., Khubchandani, J., \& Price, J. H. (2021b). The nature and extent of COVID-19 vaccination hesitancy in healthcare workers. Journal of Community Health, 46, 12441251. https://doi.org/10.1007/s10900-021-00984-3

Bueno-Notivol, J., Gracia-García, P., Olaya, B., Lasheras, I., López-Antón, R., \& Santabárbara, J. (2021). Prevalence of depression during the COVID-19
COVID-19: implicit attitudes, vaccine and well-being 
Daiana Colledani,

Pasquale Anselmi, Egidio Robusto outbreak: a meta-analysis of community-based studies. International Journal of Clinical and Health Psychology, 21, 100196. https://doi.org/10.1016/j. ijchp.2020.07.007

Chan, D. K., Zhang, C. Q., \& Weman-Josefsson, K. (2021). Why people failed to adhere to COVID-19 preventive behaviors? Perspectives from an integrated behavior change model. Infection Control and Hospital Epidemiology, 42, 375-376. https://doi. org/10.1017/ice.2020.245

Chaturvedi, K., Vishwakarma, D. K., \& Singh, N. (2021). COVID-19 and its impact on education, social life and mental health of students: a survey. Children and Youth Services Review, 121, 105866. https://doi.org/10.1016/j.childyouth.2020.105866

Chudik, A., Mohaddes, K., Pesaran, M. H., Raissi, M., \& Rebucci, A. (2020). Economic consequences of COVID-19: a counterfactual multi-country analysis. Retrieved from https://voxeu.org/article/economic-consequences-covid-19-multi-country-analysis

Colledani, D., \& Camperio Ciani, A. (2021). A worldwide internet study based on implicit association test revealed a higher prevalence of adult males' androphilia than ever reported before. The Journal of Sexual Medicine, 18, 4-16. https://doi. org/10.1016/j.jsxm.2020.09.011

Colledani, D., Anselmi, P., \& Robusto, E. (2019). Development of a new abbreviated form of the Eysenck Personality Questionnaire-Revised with multidimensional item response theory. Personality and Individual Differences, 149, 108-117. https:// doi.org/10.1016/j.paid.2019.05.044

Colledani, D., Anselmi, P., \& Robusto, E. (2021). On the association between the use of digital devices and well-being during the COVID-19 lockdown. Mankind Quarterly, 62, 255-272.

Dymecka, J., Gerymski, R., \& Machnik-Czerwik, A. (2021). Fear of COVID-19 as a buffer in the relationship between perceived stress and life satisfaction in the Polish population at the beginning of the global pandemic. Health Psychology Report, 9, 149-159. https://doi.org/10.5114/hpr.2020.102136

Epifania, O. M., Anselmi, P., \& Robusto, E. (2020). ImplicitMeasures: Compute scores for different implicit measures ( $\mathrm{R}$ package version 0.2.0). Retrieved from https://cran.r-project.org/web//packages/implicitMeasures/implicitMeasures.pdf

Fekih-Romdhane, F., Ghrissi, F., Abbassi, B., Cherif, W. $\&$ Cheour, M. (2020). Prevalence and predictors of PTSD during the COVID-19 pandemic: Findings from a Tunisian community sample. Psychiatry Research, 290, 113131. https://doi.org/10.1016/j.psychres.2020.113131

Fernández-Torres, M. J., Almansa-Martínez, A., \& Chamizo-Sánchez, R. (2021). Infodemic and fake news in Spain during the COVID-19 pandemic. International Journal of Environmental Research and Public Health, 18, 1781. https://doi.org/10.3390/ijerph18041781
Giuntella, O., Hyde, K., Saccardo, S., \& Sadoff, S. (2021). Lifestyle and mental health disruptions during COVID-19. Proceedings of the National Academy of Sciences, 118, e2016632118. https://doi.org/10.1073/ pnas. 2016632118

Glowacz, F., \& Schmits, E. (2020). Psychological distress during the COVID-19 lockdown: The young adults most at risk. Psychiatry Research, 293, 113486. https://doi.org/10.1016/j.psychres.2020.113486

Greenwald, A. G., McGhee, D. E., \& Schwartz, J. L. (1998). Measuring individual differences in implicit cognition: The implicit association test. Journal of Personality and Social Psychology, 74, 14641480. https://doi.org/10.1037/0022-3514.74.6.1464

Greenwald, A. G., Poehlman, T. A., Uhlmann, E. L., \& Banaji, M. R. (2009). Understanding and using the Implicit Association Test: III. Meta-analysis of predictive validity. Journal of Personality and Social Psychology, 97, 17-41. https://doi.org/10.1037/ a0015575

Guo, Y., Sims, O. T., Qin, W., \& Yang, F. (2021). Factors associated with symptoms of depression and psychological distress during the COVID-19 pandemic. Behavioral Sciences, 11, 13. https://doi. org/10.3390/bs 11020013

Haischer, M. H., Beilfuss, R., Hart, M. R., Opielinski, L., Wrucke, D., Zirgaitis, G., Uhrich, T. D., \& Hunter, S. K. (2020). Who is wearing a mask? Gender-, age-, and location-related differences during the COVID-19 pandemic. PLoS One, 15, e0240785. https://doi.org/10.1371/journal.pone.0240785

Haynes, B. F. (2021). A new vaccine to battle COVID-19. The New England Journal of Medicine, 384, 470-471. https://doi.org/10.1056/NEJMe 2035557

Karpinski, A., \& Hilton, J. L. (2001). Attitudes and the implicit association test. Journal of Personality and Social Psychology, 81, 774-788. https://doi. org/10.1037//0022-3514.81.5.774

Karpinski, A., \& Steinman, R. B. (2006). The single category implicit association test as a measure of implicit social cognition. Journal of Personality and Social Psychology, 91, 16-32. https://doi.org/ 10.1037/0022-3514.91.1.16

Khasawneh, M. A. (2021). The effect of the spread of the new COVID-19 on the psychological and social adaptation of families of persons with disabilities in the Kingdom of Saudi Arabia. Health Psychology Report, 9, 264-275. https://doi.org/10.5114/ hpr.2020.99003

Kilic, K., \& Marin, D. (2020). How COVID-19 is transforming the world economy. Retrieved from https:// voxeu.org/article/how-covid-19-transformingworld-economy

Kim, D. K. D., \& Kreps, G. L. (2020). An analysis of government communication in the United States during the COVID-19 pandemic: Recommendations for effective government health risk com- 
munication. World Medical \& Health Policy, 12, 398-412. https://doi.org/10.1002/wmh3.363

Kokou-Kpolou, C. K., Megalakaki, O., Laimou, D., \& Kousouri, M. (2020). Insomnia during COVID-19 pandemic and lockdown: Prevalence, severity, and associated risk factors in French population. Psychiatry Research, 290, 113128. https://doi.org/10.1016/j. psychres.2020.113128

Kose, S., Mandiracioglu, A., Sahin, S., Kaynar, T., Karbus, O., \& Ozbel, Y. (2021). Vaccine hesitancy of the COVID-19 by health care personnel. International Journal of Clinical Practice, 75, e13917. https://doi.org/10.1111/ijcp.13917

Majumdar, P., Biswas, A., \& Sahu, S. (2020). COVID-19 pandemic and lockdown: Cause of sleep disruption, depression, somatic pain, and increased screen exposure of office workers and students of India. Chronobiology International, 37, 1191-1200. https://doi.org/10.1080/07420528.2020.1786107

Mallinas, S. R., Maner, J. K., \& Plant, E. A. (2021). What factors underlie attitudes regarding protective mask use during the COVID-19 pandemic? Personality and Individual Differences, 181, 111038. https://doi.org/10.1016/j.paid.2021.111038

Marelli, S., Castelnuovo, A., Somma, A., Castronovo, V., Mombelli, S., Bottoni, D., Leitner, C., Fossati, A., \& Ferini-Strambi, L. (2021). Impact of COVID-19 lockdown on sleep quality in university students and administration staff. Journal of Neurology, 268, 8-15. https://doi.org/10.1007/s00415-020-10056-6

Merlo, E. M., Sicari, F., Frisone, F., Costa, G., Alibrandi, A., Avena, G., \& Settineri, S. (2021). Uncertainty, alexithymia, suppression and vulnerability during the COVID-19 pandemic in Italy. Health Psychology Report, 9, 169-179. https://doi.org/10.5114/hpr. 2021.104078

Moscadelli, A., Albora, G., Biamonte, M. A., Giorgetti, D., Innocenzio, M., Paoli, S., Lorini, C., Bonanni, P., \& Bonaccorsi, G. (2020). Fake news and Covid-19 in Italy: Results of a quantitative observational study. International Journal of Environmental Research and Public Health, 17, 5850. https://doi. org/10.3390/ijerph17165850

Murphy, J., Vallières, F., Bentall, R. P., Shevlin, M., McBride, O., Hartman, T. K., McKay, R., Bennett, K., Mason, L., Gibson-Miller, J., Levita, L., Martinez, A. P., Stocks, T. V. A., Karatzias T., \& Hyland, P. (2021). Psychological characteristics associated with COVID-19 vaccine hesitancy and resistance in Ireland and the United Kingdom. Nature Communications, 12, 29. https://doi.org/10.1038/s41467020-20226-9

Muthén, B. O., \& Muthén, L. K. (2012). Mplus Version 7: User's guide. Muthén \& Muthén.

Nazlı, Ş. B., Yığman, F., Sevindik, M., \& Özturan, D. D. (2021). Psychological factors affecting COVID-19 vaccine hesitancy. Irish Journal of Medical Science. https://doi.org/10.1007/s11845-021-02640-0
Nguyen, T. T. P., Nguyen, D. C., Nguyen, A. T. T., Nguyen, L. H., Vu, G. T., Nguyen, C. T., Nguyen, T. H., \& Le, H. T. (2020). Fake news affecting the adherence of national response measures during the COVID-19 lockdown period: The experience of Vietnam. Frontiers in Public Health, 8, 589872. https://doi.org/10.3389/fpubh.2020.589872

Nosek, B. A. (2007). Implicit-explicit relations. Current Directions in Psychological Science, 16, 65-69. https://doi.org/10.1111/j.1467-8721.2007.00477.x

Pappa, S., Ntella, V., Giannakas, T., Giannakoulis, V. G., Papoutsi, E., \& Katsaounou, P. (2020). Prevalence of depression, anxiety, and insomnia among healthcare workers during the COVID-19 pandemic: a systematic review and meta-analysis. Brain, Behavior, and Immunity, 88, 901-907. https:// doi.org/10.1016/j.bbi.2020.05.026

Pawliczak, R. (2021). Comparison of two currently available anti-COVID-19 vaccines. Polish Journal of Allergology, 7, 1-2. https://doi.org/10.5114/ pja.2021.102554

Radulescu, C. V., Ladaru, G. R., Burlacu, S., Constantin, F., loanăș, C., \& Petre, I. L. (2021). Impact of the COVID-19 pandemic on the Romanian labor market. Sustainability, 13, 271. https://doi.org/10.3390/ su 13010271

Ranieri, J., Guerra, F., \& Di Giacomo, D. (2021). Predictive risk factors for post-traumatic stress symptoms among nurses during the Italian acute COVID-19 outbreak. Health Psychology Report, 9, 180-185. https://doi.org/10.5114/hpr.2020.101249

Rehman, U., Shahnawaz, M. G., Khan, N. H., Kharshiing, K. D., Khursheed, M., Gupta, K., Kashyap, D., \& Uniyal, R. (2021). Depression, anxiety and stress among Indians in times of COVID-19 lockdown. Community Mental Health Journal, 57, 42-48. https://doi.org/10.1007/s10597-020-00664-x

Reno, C., Maietti, E., Fantini, M. P., Savoia, E., Manzoli, L., Montalti, M., \& Gori, D. (2021). Enhancing COVID-19 vaccines acceptance: Results from a survey on vaccine hesitancy in Northern Italy. Vaccines, 9, 378. https://doi.org/10.3390/vaccines 9040378

Richetin, J., Perugini, M., Prestwich, A., \& O’Gorman, R. (2007). The IAT as a predictor of food choice: The case of fruits versus snacks. International Journal of Psychology, 42, 166-173. https://doi.org/10.3390/ vaccines 9040378

Richetin, J., Richardson, D. S., \& Mason, G. D. (2010). Predictive validity of IAT aggressiveness in the context of provocation. Social Psychology, 41, 2734. https://doi.org/10.1027/1864-9335/a000005

Robertson, E., Reeve, K. S., Niedzwiedz, C. L., Moore, J., Blake, M., Green, M., Katikireddi, S. V., \& Benzeval, M. J. (2021). Predictors of COVID-19 vaccine hesitancy in the UK household longitudinal study. Brain, Behavior, and Immunity, 94, 41-50. https://doi.org/10.1016/j.bbi.2021.03.008
COVID-19: implicit attitudes, vaccine and well-being 
Daiana Colledani, Pasquale Anselmi, Egidio Robusto
Sallam, M. (2021). COVID-19 vaccine hesitancy worldwide: a concise systematic review of vaccine acceptance rates. Vaccines, 9, 160. https://doi.org/10.3390/ vaccines 9020160

Santabárbara, J., Lasheras, I., Lipnicki, D. M., BuenoNotivol, J., Pérez-Moreno, M., López-Antón, R., De la Cámara, C., Lobo, A., \& Gracia-García, P. (2021). Prevalence of anxiety in the COVID-19 pandemic: an updated meta-analysis of community-based studies. Progress in Neuro-Psychopharmacology and Biological Psychiatry, 109, 110207. https://doi. org/10.1016/j.pnpbp.2020.110207

Schou-Bredal, I., Grimholt, T., Bonsaksen, T., Skogstad, L., Heir, T., \& Ekeberg, Ø. (2021). Optimists' and pessimists' self-reported mental and global health during the COVID-19 pandemic in Norway. Health Psychology Report, 9, 1-9. https://doi. org/10.5114/hpr.2021.102394

Seetharaman, P. (2020). Business models shifts: Impact of COVID-19. International Journal of Information Management, 54, 102173. https://doi.org/10.1016/j. ijinfomgt.2020.102173

Steffens, M. C. (2004). Is the implicit association test immune to faking? Experimental Psychology, 51, 165-179. https://doi.org/10.1027/1618-3169.51.3.165

Tang, W., Hu, T., Hu, B., Jin, C., Wang, G., Xie, C., Chen, S., \& Xu, J. (2020). Prevalence and correlates of PTSD and depressive symptoms one month after the outbreak of the COVID-19 epidemic in a sample of home-quarantined Chinese university students. Journal of Affective Disorders, 274, 1-7. https://doi.org/10.1016/j.jad.2020.05.009

Troiano, G., \& Nardi, A. (2021). Vaccine hesitancy in the era of COVID-19. Public Health, 194, 245-251. https://doi.org/10.1016/j.puhe.2021.02.025

Türközer, H. B., \& Öngür, D. (2020). A projection for psychiatry in the post-COVID-19 era: Potential trends, challenges, and directions. Molecular Psychiatry, 25, 2214-2219. https://doi.org/10.1038/ s41380-020-0841-2

Veronese, G., Mahamid, F., Bdier, D., \& Pancake, R. (2021). Stress of COVID-19 and mental health outcomes in Palestine: The mediating role of well-being and resilience. Health Psychology Report, 9, 398-410. https://doi.org/10.5114/hpr.2021.104490

Wilczyńska, D., Li, J., Yang, Y., Fan, H., Liu, T., \& Lipowski, M. (2021). Fear of COVID-19 changes the motivation for physical activity participation: PolishChinese comparisons. Health Psychology Report, 9 138-148. https://doi.org/10.5114/hpr.2021.105007

World Health Organization (2021). WHO coronavirus (COVID-19) dashboard. Retrieved from https://covid19.who.int/

Xiong, J., Lipsitz, O., Nasri, F., Lui, L. M., Gill, H., Phan, L., Chen-Li, D., lacobucci, M., Ho, R., Majeed, A., \& McIntyre, R. S. (2020). Impact of COVID-19 pandemic on mental health in the general population: a systematic review. Journal of Affective
Disorders, 277, 55-64. https://doi.org/10.1016/j.jad. 2020.08.001

Xu, Q., Mao, Z., Wei, D., Liu, P., Fan, K., Wang, J., Wang, X., Lou, X., Lin, H., Wang, C., \& Wu, C. (2021). Prevalence and risk factors for anxiety symptoms during the outbreak of COVID-19: a large survey among 373216 junior and senior high school students in China. Journal of Affective Disorders, 288, 17-22. https://doi.org/10.1016/j.jad.2021.03.080

Yıldırım, M., \& Güler, A. (2021). Coronavirus anxiety, fear of COVID-19, hope and resilience in healthcare workers: a moderated mediation model study. Health Psychology Report, 9, 1-10. https:// doi.org/10.5114/ hpr.2021.107336

Zaami, S., Marinelli, E., \& Varì, M. R. (2020). New trends of substance abuse during COVID-19 pandemic: an international perspective. Frontiers in Psychiatry, 11, 700. https://doi.org/10.3389/fpsyt.2020.00700 